\title{
El nivel de inglés alcanzado en quinto grado de secundaria en tres colegios públicos de Lima metropolitana
}

\author{
ISABEL GARCÍA PONCE \\ Pontificia Universidad Católica del Perú - Perú \\ NORMA VECORENA SÁNCHEZ ${ }^{* *}$ \\ Universidad de San Martín de Porres - Perú \\ ELISA VELASCO ORDÓŃEZ ${ }^{* * *}$ \\ Pontificia Universidad Católica del Perú - Perú \\ Recibido el 04-09-19; primera evaluación el 08-09-19; \\ segunda evaluación el 18-08-19; aceptado el 27-08-19
}

\section{RESUMEN}

La presente investigación se enmarca en el contexto de la educación pública peruana, donde la enseñanza del idioma inglés ha sido objeto de infructuosas reformas tendientes a mejorar la situación de olvido en la que se ha encontrado por décadas.

\footnotetext{
* Doctora en Lingüística y educadora, especialista en la enseñanza de lenguas, con treinta años de experiencia docente y/o administrativa en colegios estatales, institutos superiores y la universidad y más de veinte ańos de experiencia en la formación de docentes de lengua materna y de lenguas adicionales (inglés y francés). Actualmente es profesora principal a tiempo completo y jefa del Departamento de Educación de la Pontificia Universidad Católica del Perú, donde también coordina la diplomatura de 2da especialidad en enseñanza del idioma español. Correo electrónico: igarcia@pucp.edu.pe

** Licenciada en Educación, especialidad Inglés y magíster en Investigación y Docencia Universitaria. Con estudios de especialización en la Enseñanza de Inglés en la Universidad Estatal de Nueva York en Buffalo, EE.UU. y en Saint Mary’s College en Londres, Inglaterra. Con treinta años de experiencia docente en colegios estatales y privados, institutos superiores y universidades estatales y privadas. Tiene más de veinte ańos de experiencia en formación de docentes de inglés. Ha realizado consultorías para el Ministerio de Educación del Perú (Minedu) y ha sido capacitadora en el Plan Nacional de Capacitación Docente (Plancad). Correo electrónico: normavecorena2015@gmail.com

*** Licenciada en Educación, especialidad Inglés, por la Pontificia Universidad Católica del Perú y diplomada en Estudios Avanzados en Enseñanza de Inglés por el West Sussex Institute of Higher Education, Universidad de Southampton, Reino Unido. Ha laborado como docente en el Plan Especial de Licenciatura en Educación y en el Diploma de Enseñanza de Inglés de la PUCP, en la USIL, en ESAN, en la URP y en Johns Hopkins University, Baltimore, EE.UU. Ha sido capacitadora docente en PRONAFCAP y en el CISE-PUCP. Ha realizado consultorías para Minedu, SASE Consultores, BID y otras instituciones, en diversos períodos del 2000 al presente. Correo electrónico: evelasco@pucp.edu.pe
} 
En momentos en que el Ministerio de Educación (Minedu) ha puesto en marcha una nueva política para lograr que todo egresado de la escuela pública pueda expresarse eficientemente en el idioma inglés, es oportuno conocer la eficacia de estos cambios. Mediante datos recogidos de los protagonistas, el presente estudio da cuenta de los factores que están facilitando o impidiendo su aplicación, así como de sus primeros logros, a fin de identificar elementos útiles para la toma de decisiones que aseguren la calidad de la enseñanza de inglés en los colegios públicos.

Palabras clave: enseñanza de inglés, colegios públicos, nivel de egreso, políticas educativas, percepción de la enseñanza.

\section{The level of English achieved in fifth grade of secondary school at three public schools in metropolitan Lima}

\section{Abstract}

This research is framed in the context of the Peruvian public education, where the teaching of the English language has been the objective of unsuccessful reforms aimed at improving the situation of oblivion in which it has been for decades. At a time when the Ministry of Education has launched a new policy to ensure that all students who graduate from public school can express their ideas efficiently in English, it is advisable to know the effectiveness of these changes. Using data collected from the protagonists, this study gives account of the factors that are facilitating or impeding its implementation, as well as their early successes, to identify useful elements for decisions that ensure the quality of the teaching of English in public schools.

Keywords: teaching English, public schools, graduate level, educational policies, teaching perception

\section{O nível de inglês alcançado no quinto ano do ensino médio em três escolas públicas da regiáo metropolitana de Lima}

\section{Resumo}

A presente investigação enquadra-se no contexto da educação pública peruana, onde o ensino da língua inglesa tem sido objeto de reformas infrutíferas tendentes a melhorar a situação de esquecimento em que se encontra há décadas. No momento em que o Ministério da Educação lançou uma nova política para garantir que todos os graduados em escolas públicas possam se expressar de maneira eficiente no idioma inglês, é oportuno conhecer a eficácia dessas mudanças. Utilizando dados coletados dos protagonistas, o presente estudo dá conta dos fatores que facilitam ou dificultam sua aplicação, bem como de suas primeiras conquistas, a fim de identificar elementos úteis para a tomada de decisóes que garantam a qualidade do ensino de inglês em escolas públicas.

Palavras-chave: ensino de inglês, escolas públicas, nível de graduação, políticas educacionais, percepçáo de ensino. 


\section{INTRODUCCIÓN}

La interrogante de partida de la presente investigación nace de nuestra experiencia como administradoras de pruebas de entrada en instituciones particulares de enseńanza de inglés, donde los clasificados al primer ciclo de estudios suelen proceder de colegios públicos. Verificar esta presunción y conocer sus causas dará luces para una mejor inversión del tiempo dedicado a esta enseñanza en la escuela.

La investigación es oportuna en un momento político en que el Ministerio de Educación ha lanzado el proyecto «Inglés: puertas al mundo», el cual constituye una política para el mejoramiento de la enseńanza del idioma inglés en los colegios públicos. Los cambios han empezado a darse en los colegios denominados "emblemáticos» ${ }^{1}$ sin un diagnóstico previo que se haya difundido, motivo por el cual resulta pertinente una investigación como la que presentamos aquí.

Como antecedente del presente trabajo, señalamos una investigación realizada por las autoras acerca de la enseñanza del idioma inglés en el primer año de secundaria de seis colegios públicos de la periferia de Lima. Entre las conclusiones del estudio, nos impactaron el incumplimiento del currículo oficial por los docentes, debido a considerarlo impracticable, y las limitaciones con las que trabajan, además de su poco dominio del idioma inglés, con escasas excepciones.

Son pocos y muy antiguos los estudios que hay sobre esta realidad, pero la situación descrita en ellos casi no ha variado. Para Jáuregui, Wu de Zierer y Zierer (1975), citados por Zierer-Wu (1981), las políticas emanadas del Ministerio de Educación no han dado buenos resultados, pues al ingresar a la universidad y retomar cursos de idioma inglés, los alumnos lo hacen «en calidad de principiantes, particularmente cuando se trataba de alumnos procedentes de regiones rurales». Estos investigadores han identificado las siguientes causas de este estado de cosas:

1) La escasez de profesores calificados;

2) La ausencia de una política positiva para el segundo idioma en la educación básica;

3) La falta de material didáctico para la enseñanza de idiomas; programa de recuperación (rehabilitación, remodelación y equipamiento) DV004-2009. 
4) La falta de un programa oficial debidamente elaborado para la enseñanza de idiomas;

5) Una infraestructura inapropiada;

6) La falta de apoyo administrativo en los planteles: horarios, número de alumnos por aula, etc.;

7) La falta de supervisión técnica;

8) La casi ausencia de perfeccionamiento docente;

9) El hecho de que las universidades no exploren los conocimientos de los postulantes en idioma extranjero;

10) La escasa investigación científica en la problemática de la enseñanza de idiomas;

11) La falta de cooperación entre las autoridades educacionales, los centros educativos y los centros de educación superior que forman profesores de idiomas;

12) La falta de comprensión del rol del aprendizaje de un idioma extranjero dentro de una política lingüística integral del país.

\section{MARCo TEÓRICO}

Para esta investigación, revisamos el Diseño Curricular Nacional (DCN 2009), las Orientaciones para el Trabajo Pedagógico-Idioma extranjero (2010), el Currículo Nacional de la Educación Básica de 2015, el Proyecto Educativo nacional al 2021, el Marco Común Europeo de Referencia para las Lenguas y diversas leyes y normas que han marcado la política que ha regido la enseñanza del inglés en nuestro país.

\subsection{Políticas para la enseñanza de idiomas extranjeros en la educación básica peruana}

La enseñanza de inglés no ha sido una prioridad de las políticas educativas, a pesar de que diversos estudios seńalan que el inglés se asocia con «mejores perspectivas laborales, la capacidad de comunicarse con más personas, el acceso a una gama más amplia de fuentes de información y la oportunidad de una mejor educación» (British Council, 2015, p. 57). Si bien se reconocen los intentos de mejorar la enseñanza del idioma inglés en la educación pública de nuestro país, les ha faltado continuidad y sus resultados no han tenido difusión. 
Antes de la reforma educativa del gobierno de Velasco Alvarado, la asignatura de idioma extranjero se impartía en tres horas semanales, durante los cinco años de la educación secundaria. Aparte, los colegios particulares binacionales impartían enseñanza intensiva en idiomas alemán, francés, italiano, inglés, chino y japonés con apoyo de gobiernos extranjeros. Pero el gobierno revolucionario de Velasco vio en la enseñanza de idiomas extranjeros un peligro de alienación e imposición cultural. Por ello, si bien la Ley General de Educación $^{2}$ y el Reglamento de Educación Bilingüe ${ }^{3}$ reconocían a las lenguas extranjeras como «instrumentos de cultura, de ciencia y de comunicación internacional», directivas del Ministerio de Educación ${ }^{4}$ redujeron sistemáticamente el número de horas semanales de inglés. Así, en los cuatro últimos años de la Educación Básica Regular (EBR), este número se redujo a dos, y —más adelante- en los tres últimos años,el inglés pasó a ser una de varias opciones de preparación para el mundo laboral. Al respecto, Michele Vanden Eynden (1980) precisa que esta enseńanza pasó a realizarse en «una sola clase semanal de cinco horas seguidas, horario que, si bien puede resultar razonable para una materia como costura o carpintería, resulta totalmente absurdo en el caso de la enseñanza de un idioma» (p. 181).

En los centros educativos técnicos, el número de horas semanales de inglés se redujo a uno en el último año, y se eliminó en la educación básica laboral y en los ciclos nocturnos. Estas directivas alcanzaron también a los colegios particulares donde la lengua extranjera no debía impartirse desde la educación inicial ni ser vehículo de instrucción de las demás asignaturas.

Tuvieron que pasar dos décadas para que los centros educativos de gestión no estatal bilingües y biculturales recuperaran la facultad de emplear un idioma extranjero en el desarrollo de las asignaturas de sus planes de estudios, por Resolución Ministerial 0006-94-ED 22/01/1994.

Otra novedad de la ley de 1972 (artículo 98) fue el hecho de diversificar la enseńanza de idiomas extranjeros con apoyo de las embajadas, permitiendo que los centros educativos elijan el o los idiomas a enseñar, y elaboren los objetivos y contenidos específicos de sus cursos, a partir de un programa oficial de idioma inglés para la EBR. Sin embargo, para Gutiérrez, Kong y Vanini (1980), citados por Zierer-Wu (1981), los objetivos planteados por este programa resultaron inalcanzables, debido principalmente a la escasez de personal calificado para la docencia y para realizar labores de supervisión.

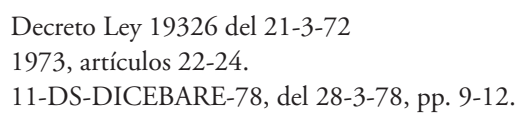


Durante el gobierno de Fernando Belaúnde Terry, la Ley General de Educación 23384 de 1982 asignó una función importante a la enseñanza del idioma inglés. En el capitulo 1, artículo 5-c de esta ley, se lee que uno de sus propósitos es «fomentar la cooperación y el intercambio con la cultura universal, especialmente la latinoamericana, manteniendo la autonomía e identidad nacional y estimulando el conocimiento de los idiomas vivos correspondientes...» (Congreso de la República del Perú, 2003). Si bien esta ley mantiene la orientación ocupacional y reivindicadora de la identidad nacional de la anterior, reconoce la importancia de abrirse a la cultura universal y al conocimiento de idiomas vivos. Por ello, el curso de inglés regresa a la EBR, pero conserva las dos horas pedagógicas semanales.

En el año 2003, la nueva Ley General de Educación 28044, como parte de una política de equidad en la educación y de protección de las lenguas nacionales (capitulo IV, artículo 20-B), «Garantiza el aprendizaje en la lengua materna de los educandos y del castellano como segunda lengua, así como el posterior aprendizaje de lenguas extranjeras» (Minedu, s.f.). Su reglamento,

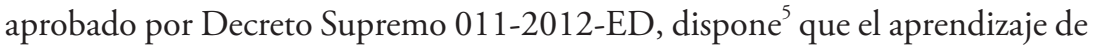
lenguas extranjeras tendrá «la finalidad de ampliar el acceso a la información y facilitar la comunicación, el conocimiento y la valoración de otras culturas y saberes» (Minedu).

El «Proyecto Educativo Nacional al 2021» del año 2007, presentado por el Consejo Nacional de Educación (2005) para cumplir con el objetivo estratégico de lograr aprendizajes pertinentes y de calidad, prevé la elaboración de un marco curricular nacional intercultural que favorezca, entre otros aprendizajes, "la capacidad de comunicarse, por diversos medios, con distintas audiencias y lenguas nacionales, empezando por la materna, y lenguas extranjeras» (p. 69).

Nace así el Diseño Curricular Nacional (2009) ${ }^{6}$, que se declara inclusivo y significativo como respuesta a nuestra diversidad sociocultural y a los retos del siglo XXl. Este diseńo plantea el desarrollo de competencias básicas durante la EBR y establece ocho propósitos de los cuales el cuarto se propone fortalecer la competencia comunicativa de los estudiantes en inglés, a fin de facilitar su acceso a la información disponible en el actual mundo globalizado.

Más adelante, el Plan Estratégico Sectorial Multianual 2012-2016, aprobado por Resolución Ministerial 058-2012-ED, compromete al sector Educación a «desarrollar capacidades que permitan que los estudiantes se inserten en el mercado laboral y enrumben el país hacia una economía de

\footnotetext{
Artículo 31, título 3, capítulo II, subcapítulo 1.

6 Aprobado por RM 0440-2008-ED.
} 
nivel global». Para ello se hacía necesaria una política que impulse cambios importantes como la enseñanza del idioma inglés desde la educación primaria y la consideración de estándares internacionales, entre otros. Sin embargo, a diferencia de los países vecinos, el interés por estos temas en nuestro país es de fecha muy reciente. Así, hasta 2012 la situación era la siguiente:

Tabla 1. Cuadro comparativo de la enseñanza del idioma inglés en América Latina

\begin{tabular}{|c|c|c|c|c|c|c|c|}
\hline Países & Argentina & Colombia & Chile & Ecuador & Uruguay & México & Perú \\
\hline $\begin{array}{l}\text { Política } \\
\text { nacional }\end{array}$ & Sí & Sí & Sí & Sí & Sí & Sí & No \\
\hline $\begin{array}{l}\text { Inglés en } \\
\text { primaria }\end{array}$ & $3 \mathrm{hrs}$ & $2 \mathrm{hrs}$ & $3 \mathrm{hrs}$ & $2 \mathrm{hrs}$ & $3 \mathrm{hrs}$ & $2 \mathrm{hrs}$ & No \\
\hline $\begin{array}{l}\text { Inglés en } \\
\text { secundaria }\end{array}$ & $3-5$ hrs & $3 \mathrm{hrs}$ & $3-4 \mathrm{hrs}$ & $3 \mathrm{hrs}$ & $3 \mathrm{hrs}$ & $3-4 \mathrm{hrs}$ & $2 \mathrm{hrs}$ \\
\hline $\begin{array}{l}\text { Referente } \\
\text { internacional }\end{array}$ & \multicolumn{6}{|c|}{$\begin{array}{c}\text { Marco Común Europeo de Referencia para las Lenguas } \\
\text { Modernas }\end{array}$} & No \\
\hline $\begin{array}{l}\text { Programa } \\
\text { nacional }\end{array}$ & $\begin{array}{c}\text { Programa } \\
\text { Nacional } \\
\text { de Inglés } \\
\text { Básico }\end{array}$ & $\begin{array}{l}\text { Colombia } \\
\text { Bilingüe }\end{array}$ & $\begin{array}{l}\text { Inglés } \\
\text { abre } \\
\text { puertas }\end{array}$ & $\begin{array}{l}\text { Vamos } \\
\text { profesores }\end{array}$ & Ceibal & $\begin{array}{l}\text { PNIEB } \\
\text { PEI }\end{array}$ & No \\
\hline $\begin{array}{l}\text { Inicia plan } \\
\text { nacional }\end{array}$ & 2010 & 2009 & 2003 & 2012 & 2012 & 2008 & $\begin{array}{l}\text { No } \\
\text { aplica }\end{array}$ \\
\hline
\end{tabular}

Fuente: Minedu, Dirección de Educación Secundaria, 2005 y 2014; y Mendoza, 2015. Elaboración propia.

En la actualidad, contamos con un documento $\operatorname{marco}^{7}$ que rige la enseñanza del inglés: Lineamientos para la implementación de la enseñanza del idioma inglés en las instituciones educativas públicas de educación básica regular. Estos lineamientos comprenden acciones centradas en fortalecer las capacidades de los profesores en el idioma inglés y aumentar las horas de clase, ya que los estudiantes «tienen como único recurso al docente de inglés en el aula, y un reducido número de horas semanales destinadas para la enseñanza del inglés (70 horas por año)». Esta situación es preocupante dado que, en los institutos de idiomas privados, ese número de horas lleva solo a concluir dos ciclos del nivel básico ${ }^{8}$. Otro inconveniente es el tema de 
la competencia de los docentes que enseñan inglés. Como se refiere en los «Lineamientos», en la evaluación a los docentes participantes en el Programa Nacional de Especialización en Inglés (2010-2011), más del 40\% fue ubicado en el nivel básico.

Otro aspecto de nuestro sistema educativo que difiere de la mayoría de países de la región es la ausencia del inglés en la educación primaria. Al respecto, en los «Lineamientos» se ha previsto incorporar esta enseńanza en los últimos años de la educación primaria pública, mediante la adquisición de destrezas básicas en entornos pedagógicos significativos.

Estos reconocimientos y compromisos dieron pie al proyecto "Inglés: puertas al mundo" (aprobado por RM 530-2015 MINEDU) sustentado en «una Política Nacional Multisectorial" que define los objetivos y lineamientos para la enseñanza y uso del idioma inglés». Se propone:

Que los beneficiarios desarrollen las competencias comunicativas del idioma inglés, para ampliar su acceso a oportunidades educativas, científicas, tecnológicas, de entretenimiento y laborales, así como para contribuir a la formación de un capital humano más productivo, y a la inserción en mercados internacionales, con miras a fortalecer la competitividad del país a nivel internacional. ${ }^{10}$

«Inglés: Puertas al Mundo» constituye un enorme salto respecto de las políticas anteriores. En el marco de estándares internacionales, plantea lograr que los egresados de la educación secundaria ostenten una competencia en idioma inglés equivalente al nivel intermedio $2^{11}$, del Marco Común Europeo de Referencia de Lenguas. Este marco, elaborado por especialistas internacionales como parte esencial de la política lingüística del Consejo de Europa, es ampliamente utilizado en varios continentes como instrumento de consulta fundamental en el campo de la enseñanza de lenguas.

Para implementar la enseñanza del idioma inglés en las instituciones educativas públicas, según los renovados parámetros, la nueva política fija los roles que deben asumir las autoridades educativas.

Al Ministerio de Educación le corresponde coordinar con otros sectores del Estado y establecer alianzas con la sociedad civil y organismos internacionales para asegurar los recursos humanos, materiales, tecnológicos y económicos que garanticen una enseñanza del inglés de calidad que satisfaga las necesidades del

\footnotetext{
9 Aprobada por Decreto Supremo 012-2015-MINEDU.

10 Recuperado de http://www.minedu.gob.pe/ingles-puertas-al-mundo p. 2.

11 El nivel intermedio del MCER corresponde al que posee el usuario independiente. Comprende dos subniveles, de los cuales el segundo (B2) corresponde al intermedio alto. Es decir, un nivel en el que uno se puede relacionar con hablantes nativos con un grado suficiente de fluidez y naturalidad.
} 
mundo laboral. Como lo indican los Lineamientos para la implementación de la enseñanza del idioma inglés en las instituciones educativas públicas de educación básica regular ${ }^{12}$ en su página nueve, el Minedu deberá prioritariamente:

1. Mejorar la competencia de los docentes en el dominio del idioma inglés, así como en enfoques metodológicos y herramientas tecnológicas para su enseñanza.

2. Brindar la asistencia técnica que requieran las instituciones educativas, Unidades de Gestión Educativa Local y Direcciones Regionales de Educación.

3. Monitorear y evaluar el desarrollo de las actividades educativas.

Aparte de efectuar las coordinaciones a nivel regional y local para el logro de los objetivos planteados, a las Direcciones Regionales y las Unidades de Gestión Educativa Local les corresponde:

4. Gestionar ante sus respectivos gobiernos regionales la asignación de los recursos presupuestales necesarios.

5. Fortalecer las capacidades y brindar asistencia técnica a los diversos actores que, a nivel regional y local, intervienen en la gestión de las escuelas donde se implemente la enseńanza del idioma inglés.

La Resolución Ministerial 199-2015-MINEDU modifica el Diseño Curricular de la Educación Básica Regular ${ }^{13}$ de 2009, presentado en el Consejo Nacional de Educación (2017), indicando competencias, capacidades y desempeños para algunas áreas curriculares, donde no se ha considerado el área de Idioma extranjero. Sin embargo, al referirse al perfil del egresado, este nuevo currículo señala la capacidad para comunicarse de forma asertiva e interactuar en diversos contextos con distintos propósitos. Esta comunicación la hará en su lengua materna o en castellano como segunda lengua, si su lengua materna es una de las 47 lenguas originarias del Perú, y en inglés como lengua extranjera.

12 Recuperado de http://www.drelm.gob.pe/drelm/wp-content/uploads/2015/06/Resolucion_Ingles. compressed.pdf

13 La Educación Básica Regular abarca los niveles de Educación Inicial (ciclos I y II, para menores de 6 años), Primaria (ciclos III, IV y V con 6 años de duración) y Secundaria (ciclos VI y VII). En este último nivel, que dura 5 años, se imparte la enseñanza del idioma inglés en los colegios públicos a razón de dos horas semanales, salvo en los colegios de jornada escolar completa, donde estas horas han aumentado a cinco. 
Las competencias en idioma inglés que el Diseño Curricular Nacional (DCN) 2016 señala para el egresado de la educación secundaria (VII ciclo) son tres. Las citamos con un resumen de la descripción de su nivel de desarrollo (pp. 51-56):

Competencia 13: Se comunica oralmente en inglés como lengua extranjera

A partir de información implícita y explícita, infiere, organiza y desarrolla ideas, extrae conclusiones e interpreta la intención de comunicación. Se adecúa a situaciones comunicativas formales e informales, expresándose con una pronunciación y entonación inteligibles, y usando recursos verbales, no verbales y para-verbales. Interactúa sobre temas habituales y evalúa las respuestas y puntos de vista escuchados para dar sus aportes.

Competencia 14: Lee diversos tipos de textos escritos en inglés como lengua extranjera.

Lee críticamente textos en inglés con algunas estructuras complejas y vocabulario variado y especializado. Integra la idea principal con información específica para construir su sentido global. Evalúa e interpreta el uso del lenguaje y los recursos textuales, así como el efecto del texto en el lector a partir de su conocimiento del contexto sociocultural.

Competencia 15: Escribe diversos tipos de texto en inglés como lengua extranjera.

Escribe textos adecuados al destinatario y al propósito de su escrito, a partir de fuentes de información variada. Organiza sus ideas alrededor de un tema central y las estructura en párrafos. Utiliza recursos cohesivos con vocabulario variado y pertinente a la temática tratada y construcciones gramaticales de mediana complejidad. Evalúa los usos del lenguaje y utiliza recursos ortográficos para mayor claridad.

Los exigentes niveles de comprensión y expresión señalados, y los roles asignados a las autoridades responsables de su cumplimiento requieren personal calificado. Todo ello revela la necesidad de apoyar la formación de profesionales en Educación con especialidad en idioma inglés. En el balance ${ }^{14}$ 2017-2018 realizado por el Consejo Nacional de Educación (2018) sobre la implementación del Currículo Nacional de Educación Básica, se indica que en 2018 fueron pocos los avances y que «en la educación secundaria no se estableció ninguna acción» (p. 18), debido a diversas circunstancias políticas, económicas y sociales ${ }^{15}$. Insiste

\footnotetext{
14 CNE 2018 - Proyecto Nacional al 2021. Balance y recomendaciones 2017-2018.

15 Huelga magisterial, cambios en la gestión ministerial, cuestionamiento curricular por el enfoque de género, fenómeno del «niño costero», entre otros.
} 
además en que el Minedu «necesita desarrollar un sistema de formación docente» en lugar de «capacitaciones aisladas» (p. 24).

Recomienda además «dar prioridad política, técnica y presupuestal a la formación inicial docente» y al «fortalecimiento de las facultades de educación de las universidades y la diversificación de especialidades según las necesidades educativas de los estudiantes». Las capacitaciones solo perpetúan acciones remediales y los docentes formados en la especialidad de inglés continúan siendo escasos.

\section{Metodología}

La investigación, de enfoque mixto, es inicialmente descriptiva, porque observa y analiza cómo se desarrolla la enseñanza del idioma inglés en los colegios públicos de la muestra. Abarca también la dimensión explicativa porque busca encontrar las razones de las situaciones que serán descritas.

\subsection{Formulación del problema}

Los cuestionamientos que nos planteamos al inicio de nuestra investigación son los siguientes:

- ¿Cuál es el nivel de inglés de los alumnos que egresan de 5to año de secundaria en los colegios públicos de Lima?

- ¿Qué factores intervienen en este resultado?

- ¿Qué percepciones de esta formación tienen los docentes y los alumnos?

- ¿Qué causas determinan los resultados de la enseñanza de inglés en dos colegios públicos de Lima metropolitana?

\subsection{Objetivos}

Los objetivos iniciales debieron reajustarse debido a que uno de los tres colegios seleccionados pasó al régimen de jornada escolar completa, con parámetros distintos en cuanto al número de horas semanales de clase y materiales de enseñanza con recursos informáticos, entre otros. Los objetivos reajustados son los siguientes:

- Conocer el nivel de conocimiento del idioma inglés con el que egresan los alumnos de dos colegios públicos emblemáticos de Lima

- Conocer las condiciones de trabajo de los docentes de idioma inglés en los dos colegios en estudio. 
- Identificar las principales causas de los resultados de la enseñanza del idioma inglés en los dos colegios del estudio.

- Averiguar si, en las actuales condiciones, las disposiciones dadas para el cumplimiento de la nueva política son factibles.

\subsection{Hipótesis}

1. Al egresar de la educación secundaria, los alumnos de los colegios públicos no muestran progresos en su conocimiento del idioma inglés.

2. La formación de los docentes de inglés que laboran en colegios públicos es insuficiente.

3. Las condiciones de trabajo en los colegios públicos no favorecen el aprendizaje del idioma inglés.

a. El número de horas de clase por semana es escaso.

b. La supervisión, el seguimiento, el acompańamiento y la capacitación a los docentes por parte de las autoridades del MINEDU es parcial o inexistente.

c. No hay coordinadores oficiales para la especialidad de inglés

4. Los objetivos planteados en el DCN son demasiado ambiciosos y los recursos disponibles son muy limitados.

Los datos fueron recogidos de las siguientes fuentes y con las siguientes herramientas:

- Revisión de documentos normativos

- Exámenes de nivel a los alumnos de 5to de secundaria de las dos IIEE seleccionadas

- Entrevistas al coordinador, al director o subdirector y a un docente por institución

- Observaciones de clases a un docente por institución

- Focus groups con los alumnos de los docentes observados

- Entrevistas a los directores de tres centros de idiomas de universidades privadas de Lima

\subsection{Población - muestra}

Se ha trabajado con los profesores y alumnos de dos secciones de quinto grado de secundaria en dos colegios públicos emblemáticos, cuyos nombres se mantienen en reserva: 


\section{I.E.E. 1}

2. I.E.E. 2

Algunos datos recogidos en el tercer colegio, que pasó a condición de jornada escolar completa, se incluyen como referencia.

\section{RESUlTADOS Y DISCUSIÓN}

Los resultados del examen de clasificación aplicado a los alumnos se analizan y explican a la luz de los documentos que norman la enseńanza del idioma inglés en los colegios públicos y de las circunstancias que enmarcan las acciones de los principales actores de la enseńanza aprendizaje.

\subsection{El nivel de inglés}

El examen de inglés aplicado a los alumnos de las instituciones educativas 1 y 2 arrojaron los siguientes resultados:

Figura 1. Nivel de inglés alcanzado al terminar la secundaria

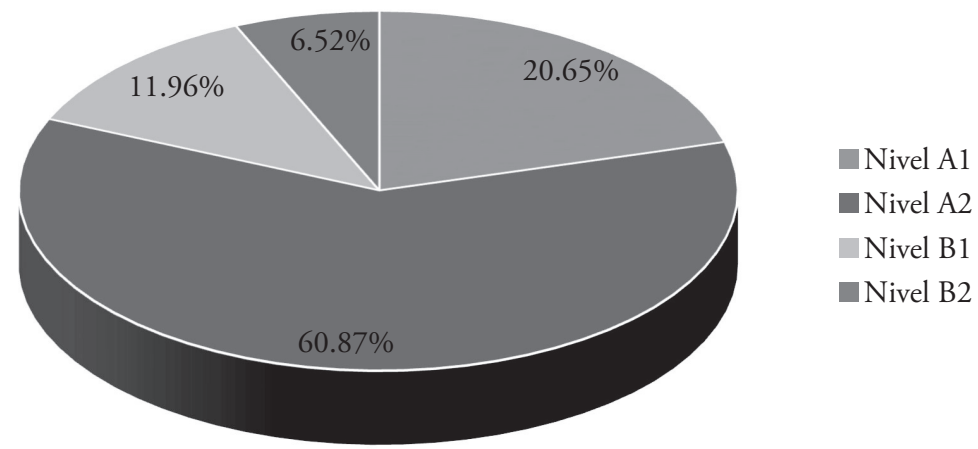

Fuente: Cronquist y Fiszbein, 2017

Más del 80\% está ubicado en los niveles básicos A1 y A2 del MCER ${ }^{16}$ (Cronquist y Fiszbein, 2017) después de cinco años de estudios con dos horas pedagógicas de clase por semana. Considerando solo ocho meses de clases, sumamos un total de 400 horas en cinco años. En este tiempo, en una academia particular, el alumno podría completar todo el nivel básico y

16 Marco Común Europeo de Referencia para las Lenguas. 
el primero o segundo de los doce ciclos del nivel intermedio. ¿Qué factores influyen para que esto no ocurra? ¿Será solo un tema de cantidad de horas de clase? Veamos la situación de las dos instituciones educativas en donde centramos nuestra investigación.

\subsection{Planificación y coordinación}

Oficialmente, esta labor está a cargo del subdirector del plantel, sea cual fuera su especialidad. En la práctica, cada institución educativa cuenta con un coordinador elegido por los docentes de la especialidad (I.E.1) o designado por el director (I.E.2).

El equipo a cargo del coordinador de la I. E.1 está conformado por cuatro docentes y el de la I.E.2 por seis. Según precisó el coordinador de esta última institución, el cargo de coordinador es solo una delegación no remunerada, lo cual reduce su autonomía y autoridad, y explica el que solo realicen reuniones «informales» y ninguna visita a clases, por temor a que los docentes «se puedan ofender». Por otro lado, tampoco hay reducción de la carga lectiva del docente coordinador por este trabajo, lo cual añade la falta de tiempo a la lista de inconvenientes para realizar una adecuada labor.

$\mathrm{Al}$ principio de cada año, los docentes de ambas instituciones se reúnen para realizar la programación anual, preparar el cartel de contenidos y las unidades a desarrollar. Asimismo, programan las reuniones que tendrán durante el año para tratar sobre el avance de las unidades, revisar materiales o compartir logros.

Los planes de trabajo son responsabilidad de cada docente y son revisados por el coordinador antes de su presentación a la subdirección. Usan como guía el DCN del 2009 dada su vigencia y que aún no hay rutas de aprendizaje $^{17}$ para inglés. En ninguna de las dos instituciones, los planes de clase son objeto de control. Otra de las dificultades que afrontan los coordinadores para realizar su labor es la falta de un horario común para reunirse como equipo, debiendo hacerlo durante el refrigerio o fuera del horario de trabajo.

Poniendo aparte las dificultades mencionadas, ambos coordinadores manifestaron que su labor les resulta gratificante. En opinión del coordinador de la I.E.1, «monitorear, apoyar a un grupo humano, intercambiar ideas con los colegas es un beneficio mutuo». Por su parte, el coordinador de la I.E.2 señaló que esta labor le ha dado la oportunidad de capacitarse en un país de habla

17 Orientaciones pedagógicas y sugerencias didácticas para una enseñanza efectiva de las competencias de las áreas curriculares de comunicación, matemática, ciencia y ambiente, personal social. 
inglesa, experiencia que le ha impregnado entusiasmo a su trabajo y el deseo de seguir actualizándose. Indicó que los progresos de sus alumnas podían verse en la feria semestral denominada "Día del logro».

Las observaciones de clase corroboraron la existencia de una planificación previa. Los docentes de ambas instituciones utilizaron una estructura coherente en sus clases y un material preparado o elegido con anticipación, como canciones, videos, material visual y ejercicios fotocopiados que se utilizaron en diferentes momentos de la sesión.

\subsection{Metodología en uso}

En ambas instituciones educativas, la metodología se señala como una carencia. Los coordinadores reconocen que falta fortalecer este aspecto en la formación de los docentes, especialmente en lo que concierne a la diversificación de los métodos y a su adecuación a los estilos de aprendizaje del alumnado.

Por su parte, las alumnas reconocen el valor del desempeño del docente en su aprendizaje del inglés y consideran «una suerte» que les toque un buen profesor. Para ellas, son buenas las clases que «involucran a las alumnas» y no son "puro texto» (I.E.2), donde los profesores «no solo hablan y hablan, sino que procuran que los alumnos entiendan» (I.E.1). En la I.E.1 indicaron que, en $3^{\circ}$ y $4^{\circ}$ ańo, la profesora faltaba demasiado, llegaba tarde y solo les ponía música o les mostraba papelógrafos. En cambio, las clases de su actual profesor «son entretenidas y sienten que aprenden». En la I.E.2, las alumnas indicaron que en $1^{\circ}$ y $2^{\circ}$ la profesora gritaba mucho y las atemorizaba, que la profesora de $3^{\circ}$ les dejaba demasiada tarea y no aprendieron nada, porque se limitaban a copiar de la pizarra. En $4^{\circ}$ y $5^{\circ}$, les cambiaron de profesora y están muy contentas con ella. Esta nueva situación favorable en ambas instituciones, probablemente se deba a los recientes programas de actualización y capacitación organizados por el Ministerio de Educación (2012), en los cuales han participado los dos docentes observados.

En cuanto al proceso didáctico de las sesiones de clase, la presentación de los temas se contextualizó teniendo en cuenta la edad de los alumnos y los lineamientos del enfoque comunicativo. Con el apoyo de ayudas visuales, auditivas, temas del momento, y la animada participación de los estudiantes, se creó un ambiente favorable para introducir los temas a estudiar, sea por su relación con los otros cursos del plan de estudios (I.E.1) o con la edad y gustos musicales de los alumnos (I.E.2). Fue un uso motivador de canciones en inglés; uno de ellos para identificar momentos históricos, y el otro como karaoke de bienvenida. 
Sin embargo, el momento que sigue es la explicación de un tema gramatical con ejemplos y una extensa sección de ejercicios mecánicos. Estos se realizan en fotocopias con una posterior corrección oral (I.E.2) o en la pizarra (I.E.1). Están ausentes las actividades comunicativas que crean la necesidad de utilizar una estructura gramatical en situaciones verosímiles que requieran su uso, o que retomen la lengua presentada en el momento previo. La sesión no está organizada en torno a una actividad de comunicación, donde todas sus partes están relacionadas significativamente y donde la transición de una a otra es fluida.

En ambas instituciones, la interacción ocurre mayormente entre el profesor y el alumno. Si bien en la I.E.2 hubo una actividad en pares, esta obedecía a intereses gramaticales.

\subsection{Evaluación y retroalimentación}

En ambas instituciones, al final de cada unidad didáctica se aplica una evaluación sumativa. Corresponde a cada profesor elaborar su proyecto y presentarlo al coordinador para proceder a un reajuste si fuera necesario. El coordinador de la I.E.1 añadió que estas pruebas son escritas y orales, y evalúan la comprensión y la producción con apoyo de audios y videos.

Durante el desarrollo de las clases, se observó que el desempeño de los alumnos en los ejercicios orales y escritos es objeto de retroalimentación inmediata, consistente en una corrección de la ortografía y la gramática más la calificación correspondiente. En la I.E.2, el coordinador informó que los docentes evalúan tareas, diálogos, posters, con apoyo de listas de cotejo y rúbricas. En el caso de exposiciones de los alumnos, la retroalimentación en cuanto a forma y contenido se intercala y luego se procede a una corrección general final. En ninguna de las observaciones se pudo apreciar espacios de autoevaluación o evaluación entre pares.

\subsection{Ambiente de clase y participación de los alumnos}

Las aulas de inglés en ambas instituciones están bien iluminadas y cuentan con carpetas individuales (I.E.2) o dobles (I.E.1) distribuidas según el patrón tradicional de filas y columnas. Los docentes cuentan con un escritorio ubicado en una esquina del aula frente a la clase y permanecen la mayor parte del tiempo en esta área, acercándose mayormente a la primera fila.

En la I.E.1, el tamaño del aula no permite una disposición diferente y los estrechos pasillos entre las carpetas no facilitan el desplazamiento del docente. En cambio, en la I.E.2, se puede circular cómodamente por el aula y las carpetas se podrían mover para los trabajos en grupo. 
Los docentes imparten instrucciones claras y usan un lenguaje empático que favorece la participación, aunque la interacción es mayormente unidireccional, docente-estudiante(s). Quienes más intervienen son las alumnas que se ubican en las primeras filas; probablemente, porque están más motivadas o porque estudian también fuera del colegio y tienen mejor nivel. El inconveniente es que los alumnos que se abstienen no son llamados y la atención del docente se centra en los que participan.

Tanto en la I.E.1 como en la I.E. 2, la clase se conduce en un ambiente agradable propicio para el aprendizaje. Los docentes tienen buena relación con los estudiantes quienes siguen sus indicaciones con interés y participan ordenada y activamente. Se aprecia un clima libre de tensiones, una actitud positiva y un trato respetuoso del docente hacia las estudiantes y viceversa. En ambas instituciones, las alumnas manifestaron que se sentían a gusto con sus actuales profesores.

En cuanto a la puntualidad, se observó flexibilidad para el ingreso de los alumnos a clase, cuyas tardanzas superaban los 30 minutos (I.E.2). Esta flexibilidad se notó también durante la sesión, pues los alumnos entraban y salían del salón libremente. Cabe preguntarse si esta situación incide en su aprendizaje del idioma.

Durante el desarrollo de las clases, la lengua de enseñanza fue siempre el inglés. Las alumnas reforzaron este hecho, precisando que los docentes recurren al castellano cuando notan que los alumnos no han entendido. Los trabajos y los exámenes los presentan en inglés y las preguntas en clase tratan de formularlas en este idioma, pero también lo hacen en castellano. El nivel de desempeño de las alumnas y el tipo de actividad determinan la flexibilidad que se da a la exigencia del uso del inglés. Por ejemplo, en la I.E.1 se observó que las preguntas de las alumnas eran respondidas en la lengua en que habían sido formuladas. En ocasiones (I.E.2), se trabaja en inglés la comprensión lectora y se enlaza con un debate en español.

\subsection{Materiales y equipos}

Las dos instituciones educativas cuentan con los libros y cuadernos de trabajo del Minedu, aunque solo una de ellas (I.E.1) también recibe los CD; y las fichas que años atrás se elaboraron como apoyo al texto, no se conocen. Los directivos manifestaron que estos materiales llegan y son distribuidos a tiempo a docentes y alumnos. Sin embargo, los coordinadores los encuentran muy recargados de unidades y, como son prestados, no se pueden marcar. En la I.E.1, los profesores reunidos deciden qué unidades van a desarrollar y en la I.E.2, no lo 
utilizan, porque, además, no concuerdan con el DCN. Las alumnas de la I.E.1 mencionaron que «el libro es bueno, pero más avanzado» y, las de la I.E.2, que el libro «no se usó». Durante las observaciones, se pudo apreciar que a los profesores les resultaba práctico trabajar con fotocopias.

Si bien la I.E.2 dispone de un "aula de inglés» bien equipada y ambientada, no es suficiente y ambas instituciones esperan que, al pasar al horario de jornada completa, se mejoren los equipos y demás recursos para la enseñanza del idioma inglés.

Los materiales de más aceptación por parte de los alumnos son los videos, los CD, las figuras, la grabadora, el televisor del aula de inglés, las separatas y fotocopias. Con ellos elaboran afiches, leen cuentos, hacen viñetas, cantan, presentan exposiciones y hablan sobre ellas mismas. Las actividades que más disfrutan son los trabajos grupales, la preparación y exhibición de menús, la exposición de temas, y salir a la pizarra. Preguntaban sobre lo que eran capaces de hacer en inglés y respondieron que podían cantar, saludar, presentarse, preguntar el nombre de una persona o dónde se encuentra, averiguar una dirección, pedir comida en un restaurante, expresar lo que les gusta o no les gusta. Todo ello da cuenta de un buen uso del enfoque comunicativo.

\subsection{Formación y capacitación docente}

Ambos docentes observados se dirigen a sus alumnas en inglés. El docente de la I.E.1 tiene buen dominio del idioma y posee un F.C.E. ${ }^{18}$. Durante el desarrollo de la sesión presenta el tema, da explicaciones y ejemplos siempre en inglés. El docente de la I.E.2 es de la especialidad de Lengua y Literatura, pero declara tener un nivel intermedio de inglés y haberse capacitado dos meses en un país de habla inglesa. En las observaciones de clase se pudo apreciar que la docente da instrucciones, conduce las actividades y retroalimenta las participaciones de sus alumnos en inglés. Su gramática, vocabulario y pronunciación son adecuados y está muy motivada para continuar mejorando.

Uno de los directivos entrevistados (I.E.2) manifestó que a nivel de la UGEL hay escasez de docentes titulados de la especialidad de inglés y que en su I.E. completan el equipo con profesores de otras especialidades que tengan estudios de inglés en institutos.

En cuanto a las capacitaciones, ambos coordinadores manifestaron que no son frecuentes y que últimamente no ha habido ninguna. El coordinador de la

18 First Certificate in English. Examen de inglés de Cambridge, correspondiente a un nivel intermedio alto. 
I.E.1 manifestó que estas tratan más sobre el mejoramiento de la lengua que sobre metodología. La coordinadora de inglés de la IE 2 expresó que cuando hay capacitaciones los docentes no siempre van, porque son renuentes a asumir cambios.

\subsection{Expectativas, proyecciones y utilidad del curso de inglés}

Las dos instituciones educativas están próximas a convertirse en centros de jornada escolar completa. Ello significa que habrá cambios en cuanto a las exigencias pedagógicas a los docentes, quienes deberán innovar y asumir nuevos retos; por ejemplo, trabajar con las estrategias de las rutas de aprendizaje. En la IE 2 están a la espera de la capacitación para directivos y para el personal en general, ya que en marzo de 2016 empezarán a funcionar con horario de jornada completa y cinco horas de inglés a la semana. Los docentes sí están siendo capacitados para esta conversión.

Por su parte, los docentes apreciarían que quien realice la labor de monitoreo sea un profesor de la especialidad de inglés. Actualmente, esta tarea la realizan los subdirectores, pero aparte de la información que transmite a los docentes sobre las capacitaciones que ofrece el Minedu, no se aprecia mayor apoyo para el curso de inglés. Desde la UGEL ${ }^{19}$, no hay especialistas que acompañen el desempeño de los docentes de inglés (I.E.2). El coordinador de la I.E.1 manifestó que, por el hecho de ser colegios emblemáticos, ya ha habido un cambio favorable, pues ahora tienen un promedio de 25 alumnos por aula que es la mitad de los que tenían antes. A pesar de ser instituciones emblemáticas, según mencionaron los directivos entrevistados, no ha habido cambios ni en los materiales de enseñanza ni en lo pedagógico. Eso sí, expresaron que los docentes tienen un mayor compromiso para asumir responsabilidades para el cumplimiento de las normas y proyectos del Minedu, así como de su plan anual de trabajo. El coordinador de la I.E. 2 expresó que gracias al apoyo de su institución pudo implementar el aula de inglés, de cuyo buen uso pudimos dar fe durante las observaciones de clase.

Las alumnas de la I.E.1 opinaron que al inglés no se le daba la debida importancia, que todo dependía de los profesores y que el Ministerio debería visitar los colegios. Para las alumnas de ambas instituciones, aprender inglés les servirá para seguir una carrera universitaria y mejorar sus posibilidades laborales. También reconocen que es un idioma universal, que les servirá para comunicarse con personas extranjeras (I.E.1) y que saber inglés es una base para aprender más y para hacer traducciones (I.E.2).

19 UGEL= Unidad de Gestión Educativa Local 
En la tercera institución visitada (I.E.3) que —al momento de la entrevista— ya se había convertido en centro de jornada escolar completa (JEC), el coordinador nos indicó que había dificultad de conseguir profesores idóneos por el aumento de horas de clase de inglés de 2 a 5 por semana. Habría entonces un problema que resolver previo al aumento de horas en los colegios públicos y a la ampliación de esta enseñanza a los últimos años de la educación primaria.

\subsection{Situación de los egresados de escuelas públicas al ingresar a la universidad}

Podemos preguntarnos ahora qué retos enfrentan los egresados de escuelas públicas al ingresar a la universidad; especialmente si eligen instituciones privadas, cuyas exigencias en cuanto al conocimiento de idiomas empiezan desde el pregrado. Para tener un mayor acercamiento a este tema, entrevistamos a las directoras de los centros de idiomas de tres universidades privadas de Lima que reciben alumnos egresados de escuelas públicas y que acceden a dichas universidades mediante becas del gobierno para alumnos destacados. Las tres universidades tienen requisitos en cuanto al conocimiento del idioma inglés al concluir los cinco ańos de formación profesional.

Siendo esta exigencia facultativa en el pregrado, cada universidad pone sus reglas. En la universidad 1, el primer control se realiza al completar los dos años de estudios generales, momento en que se debe acreditar avances a razón de un curso de inglés por ciclo, de los seis que se exigen al finalizar el pregrado. En esta etapa, la universidad 2 exige el nivel intermedio (B2) y la universidad 3, el nivel básico (A2).

Las tres directoras coincidieron en manifestar que la mayoría de los alumnos procedentes de escuelas públicas, ingresan con un nivel «elemental, bajo o nulo» de conocimiento de inglés y, al ser evaluados, van clasificados al nivel inicial.

En la universidad 1, estos alumnos postergan su avance del inglés porque tienen bajo rendimiento también en los otros cursos y tienen temor de perder la beca por bajo rendimiento. Otro problema de estos alumnos es su dificultad para participar en clase e incluso para expresarse en castellano, además de otras habilidades blandas. Su porcentaje de repitencia es de un $40 \%$, motivo por el cual la universidad les ofrece cursos de nivelación y talleres de reforzamiento los domingos.

En la universidad 2, el apoyo que reciben estos alumnos se reduce a lo económico, ya que "gozan de un significativo descuento». En esta universidad, los alumnos avanzan los ciclos de inglés a la par de sus estudios universitarios. 
En la universidad 3, estos alumnos tienen dificultad para comunicarse oralmente y por escrito, y son incluidos en programas de tutoría de una hora semanal de inglés como refuerzo, con el apoyo de una tutora practicante y alumnos voluntarios de los cursos avanzados. El índice de repitencia ha mejorado a partir del aumento de la duración de los ciclos. Los alumnos de Beca Vocación Maestro deben acreditar el nivel B2 como parte de su programa. Los demás alumnos becarios siguen la exigencia general (A2).

Por otro lado, la oficina de becas ofrece la asistencia de psicólogos para tratar temas personales que incidan en el rendimiento académico de estos alumnos. En sus pensiones, gozan de un descuento del 25\%.

En relación con la problemática mencionada, las tres directoras manifestaron que, a juzgar por lo observado en estos alumnos escogidos entre los mejores de sus colegios, la enseñanza de inglés en la escuela pública tiene serias deficiencias.

En la universidad 1, la directora del Centro de idiomas señaló como prioritarios los siguientes problemas: el bajo nivel de inglés de los docentes, la falta de profesores especialistas en la enseñanza del inglés, la sobrepoblación de alumnos por aula, el insuficiente número de horas de clase, el poco acceso a materiales de calidad por su alto costo, la falta de contacto del alumno con el idioma inglés fuera del salón de clase. Agregó que, a nivel familiar, estos alumnos son la primera generación con educación superior.

Para la directora del Centro de Idiomas de la universidad 2, en las escuelas públicas falta una buena enseñanza en el idioma, lo cual se agrava con muy pocas horas semanales de clase.

La directora del Centro de Idiomas de la universidad 3, quien manifestó haber recogido estas apreciaciones de los alumnos, indicó que los profesores repiten lo mismo todos los años, solo enseñan un vocabulario básico, no usan libros, y se limitan a hacer copiar lo que escriben en la pizarra. Como opinión personal ańadió que las capacitaciones de los docentes no son suficientes, ni cuentan con una política de colaboración para difundir lo aprendido. Asimismo, los programas de inglés no se ejecutan de forma coherente y con una progresión clara. Mucho se ha enfatizado en el aumento de horas de clase, pero no se ha previsto lo que esto implica.

En resumen, las tres directoras entrevistadas coincidieron en opinar que los alumnos egresados de escuelas públicas, al ingresar a la universidad, presentan un bajo nivel de conocimientos del idioma inglés y requieren mucho apoyo. 


\section{Conclusiones}

Al concluir el presente estudio, es oportuno señalar los elementos identificados como factores que obstaculizan o facilitan la aplicación de la nueva política, a fin de contribuir a la toma de decisiones que aseguren la calidad de la enseñanza de inglés en los colegios públicos.

1. Los alumnos que egresan de la educación secundaria pública alcanzan solo un nivel incipiente de conocimiento del idioma inglés, aún en los colegios «emblemáticos» de Lima.

2. La ausencia de reglamentos y acciones previas que hagan factible la aplicación de las nuevas políticas con respecto a la asignatura de inglés en la educación básica traban su eficacia.

3. Hace falta escuchar la voz de los docentes respecto del material didáctico que distribuye el Minedu y adaptarlos de acuerdo a sus requerimientos para asegurar su uso.

4. Dado el perfil de los docentes de inglés de los colegios públicos, es necesario acompañarlos en su desempeño pedagógico y fortalecer sus habilidades en el idioma que enseñan.

5. El problema de la escasez de profesores de idiomas calificados se hace más grave con el aumento de horas de clase de inglés por semana que propone la nueva política.

6. Si bien las capacitaciones de docentes han sido efectivas en las dos instituciones del estudio, no dejan de ser casos individuales. No se registran iniciativas de apoyo a la formación profesional de estos especialistas en las Facultades de Educación.

7. La nueva política para la enseñanza del idioma inglés en colegios públicos reconoce su valor en la formación de los educandos, pero el cambio requiere mucho más que capacitaciones o distribución de materiales.

\section{REFERENCIAS BIBLIOGRÁFICAS}

British Council (2015). English in Peru. An examination of policy, perceptions and influencing factors. Recuperado de https://www.britishcouncil.pe/sites/default/ files/english_in_peru_may_2015.pdf y http://www2.congreso.gob.pe/Sicr/ CenDocBib/con4_uibd.nsf/\$ViewTemplate\%20for\%20Documentos?Ope nForm\&Db=0C4E41B6415D91AF05257F3E005ECB39\&View=yyy 
Congreso de la República del Perú (s.f). Ley General de Educación. Ley 23384. Capítulo I de los Fundamentos de la Educación. Artículo 5. Recuperado de http://www4.congreso.gob.pe/comisiones/1998/educacion/ lgendu/cap1.htm

Consejo Nacional de Educación (2005). Hacia un Proyecto Educativo nacional 2006-2021. Lima: Consejo Nacional de Educación. Recuperado de http:// www.cne.gob.pe/uploads/proyecto-educativo/versiones/encarte-hacia-unproyecto-educativo-nacional.pdf

Consejo Nacional de Educación (2017). Revisión de las Políticas Educativas 2000-2015. Lima: Unesco Lima. Recuperado de http://www.cne.gob.pe/ uploads/revision-politicas-educativas-2000-2015.pdf

Consejo Nacional de Educación (2018) Proyecto de Educación nacional al 2021. Balance y recomendaciones 2017-2018. Recuperado de http://www.cne.gob. pe/uploads/publicaciones/publicacion/2018/balance-pen-2017-2018.pdf

Cronquist, K. y Fiszbein, A. (2017) El aprendizaje del inglés en América Latina (2017). El Diálogo. Liderazgo para las Américas. Recuperado de https:// www.thedialogue.org/wp-content/uploads/2017/09/El-aprendizaje-delingl\%C3\%A9s-en-Am\%C3\%A9rica-Latina-1.pdf

Ministerio de Educación - Minedu (2003) Ley General de Educación. Ley 28044. Artículo 20: Educación Bilingüe Intercultural. Recuperado de http:// www.minedu.gob.pe/p/ley_general_de_educacion_28044.pdf

Ministerio de Educación - Minedu (2005). Plan Nacional de Educación para Todos 2005-2015, Perú. Hacia una educación de calidad con equidad. Ministerio de Educación. Foro Nacional de Educación para todos. Lima: Minedu.

Ministerio de Educación - Minedu (2012). Marco de Buen Desempeño Docente. Un buen maestro cambia tu vida. Lima: Ministerio de Educación

Ministerio de Educación - Minedu (2014) Lineamientos para la implementación de la enseñanza del idioma inglés en las instituciones educativas públicas de educación básica regular. Recuperado de http://www.drelm.gob.pe/drelm/ wp-content/uploads/2015/06/Resolucion_Ingles.compressed.pdf

Ministerio de Educación - Minedu (2015). Inglés, puertas al mundo. Recuperado de http://www.minedu.gob.pe/ingles-puertas-al-mundo/

Mendoza, María de los Ángeles (2015). English Teaching as a Second Language Educational Public Policies. Brief Comparative Study in Colombia, Costa Rica, Mexico and Uruguay. Revista Latinoamericana de Educación Comparada, 6(8), 123-135.

Vanden Eynden, Michele (1980). Enseñanza de idiomas en la educación peruana. Lexis, 4(2), 179-189.

Zierer-Wu, Carolina (1981). La situación de la enseñanza de idiomas en el Perú. Boletín AEPE No 25. Biblioteca del profesor. Centro Virtual Cervantes. Recuperado de https://cvc.cervantes.es/ensenanza/biblioteca_ele/aepe/pdf/ boletin_25_14_81/boletin_25_14_81_08.pdf 